\title{
A software system for real-time control of psychological experiments
}

\author{
JOSEPH W. TERNES, RONALD EHRMAN, and CHARLES P. O'BRIEN \\ Department of Psychiatry, University of Pennsylvania, Philadelphia, Pennsylvania 19104, and \\ Philadelphia Veterans Administration Medical Center, Philadelphia, Pennsylvania 19104
}

\begin{abstract}
This paper describes an all-purpose experimental system, "APES," for use in a microprocessorcontrolled behavioral pharmacology laboratory. APES is an assembly language program that can run on any of the DEC PDP-11 family processors under an RT-11 single-job operating system. Its main purpose is the real-time control of psychological experimentation. The capabilities of the system are: (1) system generation of all operant or Pavlovian conditioning paradigms, (2) collection and storage of both behavioral and physiological data in a machinereadable format for later statistical analysis, and (3) operation that can be accomplished by individuals who have no computer programming experience.
\end{abstract}

This paper describes an operating system for an animal behavioral pharmacology laboratory that studies both conditioned and unconditioned responses to pharmacological agents. The approach employs multiple dependent measures, such as operant behavior and physiological variables. Since the system is broadly applicable to most areas within experimental and physiological psychology, we refer to it as the "all-purpose experimental system," or APES.

Formerly, a laboratory of this type would have been built in a very different way. Behavioral schedules would have been created by connecting combinations of electromechanical or solid state function modules, such as AND gates, timers, and counters. With these, it is possible to implement any kind of Pavlovian or operant conditioning schedule. Additional versatility would have been added by the use of plugboards that would allow experimenters to alternate between different schedules by replacing one plugboard with another. Physiological data would have been collected on polygraphs and strip chart recorders. Physiological data reduction would have involved digitizing analog data by hand and then typing these data into a computer. Reduction of behavioral data would have consisted of copying numbers from various counters into a log book and subsequently typing them into a computer. Now, however, the availability of cheap and precise analog-to-digital (A/D) converters and automated data processors such as the LSI-11 microprocessor have made it feasible to develop a computer-controlled laboratory. APES is an operating system for such a laboratory.

APES is an assembly language program that can run on any of the DEC PDP-11 family processors under an RT-11 single-job operating system. It was developed

This project was supported by Veterans Administration Medical Research Grants to J. W. Ternes and by NIDA Grants 01218 and 00586 to C. P. O'Brien. by psychologists at the Center for Drug Research and Treatment at the Philadelphia VA Medical Center for a program of animal experiments involving Pavlovian conditioning and the role of opponent processes in drug addiction. It was designed with generalization and portability in mind. Thus, there are a number of parameters and variables built in that simplify the task of adapting it to other environments.

The features of the system are as follows. (1) The system is interactive and devised to allow its use by people who have no computer programming experience. (2) The system is able to simultaneously control operant conditioning schedules, drug delivery on fixed schedules, and collection of physiological data such as heart rate, respiration, and skin temperature. (3) The conditioning schedules are flexible enough to allow for any kind of operant or Pavlovian conditioning paradigm. This includes accurate timing and provisions for handling signals both entering (responses) and leaving (stimuli) the computer. (4) The system is able to operate at least eight independent and unrelated schedules in eight different experimental chambers simultaneously. This means that they are independently programmable and their execution proceeds independently of events in other schedules. (5) The system has a flexible scheme for storing data in a machine-readable format for later statistical analysis or graph construction. The data include, at the discretion of the operator, any or all of the following: (a) physiological variables, including heart rate, respiration, and skin temperature, with room for expansion to other variables; (b) the values on incremental counters at various times; (c) the times at which the schedules present stimuli and reinforcers (e.g., buzzer, food, drug infusion); and (d) the times at which the subjects respond (e.g., lever and button actuation). (6) The system is able to operate continuously for long periods of time (i.e., 6 weeks). This means that it is possible to create, debug, store, and retrieve 
schedules while other schedules are running and collecting data. Also, because of limited disk space, collected data are stored as compactly as possible.

Presently, the system runs on an LSI-11 microcomputer and interfaces through commercially available voltage converters, power drivers, and debouncing switch inputs to any combination of eight primate or rodent conditioning apparatuses.

\section{OVERVIEW OF “APES”}

APES operates as a software simulation of electromechanical function modules.

In general, every schedule is made up of two parts: a wire list and a configuration. The configuration is simply a list of the modules needed for the schedule, and the wire list is a list of connections between modules. A special module is provided for interfacing with the outside world. This module, called the SYSTEM MODULE, is a representation of the environment or test apparatus that houses the subject during an experimental session. An output from it is equivalent to an output from an experimental chamber such as a Skinner box (e.g., a leverpress). An input to it is equivalent to an input to the box (e.g., a tone is activated).

The user gives each configuration, wire list, and module within a configuration a nine-character name. Since there is never ambiguity as to whether the user is referring to a configuration, wire list, or module, names need not be unique among these entities.

Both configurations and wire lists can be stored in a library. The library is capable of storing up to 256 different wire lists for each configuration. Consistent with the plugboard analogy, in which several plugboards may be applied to the same setup of modules, several wire lists can be used with the same configuration. The maximum number of configurations that can be stored is 256 .

There are two areas of memory that the user is concerned with. The first is the "work area." This lies physically at the end of all code, but below the resident portion of the operating system. All transfers to and from the "library" and "box area" originate or terminate here. All schedule creation, editing, and displaying takes place here. This area also has other purposes, but they are transparent to the user.

The second area is the box area. This is where schedules reside when they are running. It is divided into sections. The number of sections is a parameter, normally set to the number of experimental chambers being controlled. The researchers will generally assign numbers to the experimental chambers and let each one correspond to a section.

A schedule is never connected to the outside world until the user loads it into a section. At that time, the program asks which pins on the hardware parallel interfaces are to be logically connected to which inputs and outputs on the system module. Thus, the same schedule can be loaded into several different sections, with each section's system module associated with different interface positions.

A special module, called the "PHYSIO module," is provided. The user can include this module in his schedule and make use of the behavioral timing facilities to turn physiological data collection on and off. This makes it possible to automatically take measurements at regular intervals, for example, for $10 \mathrm{~min}$ every $3 \mathrm{~h}$, with no operator interaction.

Physiological data collection can also occur independently of the behavioral schedules. The three measures currently available are skin temperature, respiration, and heart rate. These are examined five times each $1 \mathrm{sec}$, averaged over a period of $15 \mathrm{sec}$, and stored in the $\log$ file. The entire arrangement is parameterized, so that it is easy to modify the sample rate, storage interval, and number of channels in use.

Users typically assign one set of physiological variables to each experimental chamber. However, these assignments are also parameterized, and they are therefore simple to modify. Commands are provided to start and stop data collection for individual boxes or for a series of boxes simultaneously.

The LSI-11 system on which APES was developed has a dual RK-05 disk subsystem. These are 2.5-MB hard disks, one of which is fixed and the other removable. The configuration and wire list library, along with the RT-11 operating system and the APES program itself, reside on the fixed platter. A log file, named by the user, resides on the removable disk. A log file is opened upon request when the user enters APES and is closed automatically when he exits.

The $\log$ file compactly stores various types of information, including all physiological and behavioral data. In addition, the important information about any commands that affect the box areas are stored here when they are issued. For example, it is possible to determine which schedules were loaded into specific experimental chambers, the time and date each behavioral schedule was started and stopped, and when physiological data collection began and ended.

By examining the log file at a later time, one may perform various types of statistical analyses, such as interresponse time distributions as a function of stimuli presented, or correlations between stimuli and physiological measures. A FORTRAN-callable assembly language subroutine facilitates extracting and unpacking data from the log file.

\section{CIRCUIT SIMULATION AND EXECUTION}

The heart of the behavioral part of APES is the simulation of digital circuits. This section discusses the propagation of signals within a circuit.

In an actual working digital circuit, signals propagate 
in parallel. An output changes state, and all the inputs connected to it receive the change at more or less the same time. Unfortunately, true parallel processing is not possible with a single CPU. Therefore, a recursive routine, PRPGAT was introduced to propagate signals serially.

For example, assume an output on Module A changes state. That output is connected to inputs on Modules B and $C$. Changing the state of the input on Module B causes two of its outputs to change. Which path is to be followed first, the outputs of Module B or the input to Module C? This is a tree situation. The question amounts to whether we scan its depth or its width first. We decided on the depth-first scheme because it involved simpler code and a much shallower stack. Thus, we follow the outputs of Module B before sending Module A's output to Module C.

The procedure is as follows: PRPGAT is called with the module number, a pointer to that module's module data area (MDA), and a new 16-bit output image. The output image is a representation of all the module's outputs, beginning with Bit 0 and ending with Bit $n-1$, where $\mathrm{n}$ is the number of outputs. The remaining $16-n$ bits are zero. PRPGAT compares this new image with the old image stored in the second word of the MDA and generates a map of the bits that changed. It then copies the new output image into the MDA, replacing the old image but keeping the change map.

Each output pin is connected to a chain of inputs in the wire list, all of which must receive the new state information. So, for each output that changed, and for each input connected to that output, PRPGAT determines the module number, type, and MDA pointer. With this information, plus the input pin number and new value, it calls the module service routine of that type of module. It is the job of that routine to update its own input image in its MDA and return its own new output image. PRPGAT then calls itself with this information. Notice that the module service routine determines new outputs as a function of new inputs. This is where the function of a module is defined.

Recursion will end when a node is encountered that does not generate new branches. This can happen in any of three ways. First, if an input transition does not cause a change in any of the module's outputs, the module service routine will return a new output image that is identical to its old one. Therefore, the change map will contain all zeros, and no new signals are generated. Second, if an output changes but there are no inputs connected to it, recursion will end. Third, if an input to the SYSTEM module changes and the appropriate signal is sent to the experimental chamber, recursion will end. In this case, the SYSTEM module service routine always returns a new output image identical to its old one, because the SYSTEM module outputs (outputs from the experimental apparatus) are determined by experimental subjects only.

\section{SIGNAL INITIATION}

A signal is initiated into a schedule in one of three ways: An animal in an experimental chamber makes a response, a timing request is satisfied, or a user issues a command. There are three common points that should be mentioned.

First, whenever a signal is actually being transmitted, the processor must be at a high enough priority so that it cannot be interrupted by the initiation of another signal. For example, if an interrupt does come during this time, it will be delayed until the priority comes down again. Unfortunately, the LSI-11 processor only has two levels of priority: high and low. Problems are sometimes encountered when using the RT-11 input/ output routines, which do not respect priorities as much as they might on a different CPU.

Second, signal initiation occurs when the PRPGAT routine is called at next Level 0 (i.e., the root of the propagation tree). Any routine that does this must also place a return address in the location SPSAVE in case the stack overflows. The stack can overflow either because a propagation tree is too deep (not likely) or because an oscillating circuit has been triggered. If this happens, PRPGAT stops the box containing the oscillating circuit, gives the user a message, and returns to the location stored at SPSAVE.

Third, signals are always triggered by some kind of event. Each event carries with it information identifying the experimental chamber number to which it applies. An event cannot occur when another is propagating, either in the same chamber or in a different one. When no signals are being propagated, APES attends to its user interface, the command service routines. Thus, as in any parallel processing single-CPU system, concurrency is merely an illusion to the user.

There are four commands that cause a signal to be initiated. They are LOAD BOX, RUN, INIT, and MANUAL. Of these, only MANUAL needs further elaboration. The MANUAL command allows the user to alter the value on an unconnected input of any module in a schedule that is in a section. The command inputs the module and pin names, calls the appropriate module service routine, and then calls PRPGAT at next Level 0 with the new output image.

\section{PARALLEL INTERFACE INPUTS}

When an animal presses a lever, 1 bit in the input buffer of one parallel interface (DRV-11) changes from zero to one. When the animal releases the lever, the bit changes back to zero. APES detects each of these changes, and, if the interface bit in question is assigned to an output of the SYSTEM module of a running schedule, it will propagate that transition. Through the use of some special-purpose circuitry outside the com- 
puter, each transition generates an interrupt. Each DRV-11 has its own interrupt service routine (called DRV1IN, DRV2IN, etc.) whose purpose it is to copy the input buffer into a register and pass that, along with some identifying information, to the DRVSRV routine. DRVSRV creates a copy of the SYSTEM module output image for each running box in a scratch array called SYOIMG. Then, by indexing into the OUTTAB table, it determines which box and SYSTEM module output pin are assigned to each bit on the interrupting interface and sets or clears that bit in the SYOIMG table.

SYOIMG is now a table of the NEW output images of every box's SYSTEM module. Note that, in most cases, only 1 bit in the whole array will have changed. The routine calls PRPGAT once for each work in the array (that is, for each schedule) to propagate the signals. When the count reaches zero, KWVSRV calls the time-out routine. This is often just another entry point to the module service routine. There, the module can change its outputs as required.

\section{PHYSIOLOGICAL DATA COLLECTION}

In the specific environment for which APES was designed, six primate test apparatuses were to be operated simultaneously. The researchers wanted to collect physiological information such as the skin temperature, respiration, and heart rate for each animal. However, there was only one 16 -channel A/D converter. In order to accommodate this disparity in numbers, a certain degree of flexibility was required. This was implemented by the use of several tables.

To begin, physiological channels are grouped contiguously into "physiological data sets." Each set contains all three variables. The number of sets is parameter NPHYS, which is set to four in accordance with the current wiring. Another parameter, PHYLEN, determines the number of variables in each data set. This is set to three. Two tables, PHYSET and PHYBOX, list the set number as a function of the section number and section number as a function of set number, respectively. Since there are now fewer sets than there are sections, PHYSET will obviously have some nil entries. Another table PHYSRV, contains pointers to the channel service routines associated with each channel of the A/D converter. This effectively defines the type of variable on that channel.

How and when are these A/D channels examined? Every $200 \mathrm{msec}$, a time queue entry times out and jumps to the PHYINT routine. This interval is known as the sampling period and is given by the SAMPRD parameter. PHYINT cycles through the 16 channels, reads the data on each one, and passes them to the appropriate channel service routine as listed in the PHYSRV array.

The three channel service routines are TEMP, RESP, and HEART. These have the job of updating two arrays:
PHYSUM and PHYONT. PHYSUM is a double-work array that contains a summation of measurements as a function of channel number. PHYONT is a parallel array that contains the number of measurements that have been added to each element of PHYSUM. The TEMP routine simply adds the $A / D$ reading to the PHYSUM entry and increments the corresponding PHYONT value.

RESP has a more difficult job. Respiration is measured by a strain gauge, a thin elastic tube filled with mercury and set around the monkey's chest. When the monkey inhales, the tube stretches, and the electrical resistance through it increases. When he exhales, the resistance decreases again. It is the voltage across this strain gauge that is read by the A/D converter. The RESP routine receives this reading. By comparing it with two previous values, RESP is able to determine whether the slope of the voltage curve has gone from positive to negative. When it does, RESP adds the elapsed time since the last such peak to the PHYSUM array entry and increments the corresponding PHYCNT entry. Thus, respiration is measured in terms of elapsed time between maximum inhalation.

HEART is similar to RESP in that it measures elapsed times. However, because the technology is more standard, and because the period involved is much shorter, a positive-going zero crossing of the channel reading is used to determine the end of a cycle.

Notice that the data arrays are designed such that at any time, an average reading can be obtained by divid. ing a PHYSUM element by its corresponding PHYCNT element. This is what PHYINT does to each box after $15 \mathrm{sec}$ of sampling has passed. This $15-\mathrm{sec}$ interval is called the storage period. It is defined by the STRPRD parameter.

When the storage period has elapsed for a given box, PHYINT obtains this average for each channel in the data set associated with that box. Then for each channel in the set, it compares the average with the corresponding value last stored in the log file. If the difference is greater than a certain noise tolerance, it stores the new average in the $\log$. The noise tolerance is given by the PHYTOL array as a function of the type of variable in question. Whenever a variance is stored in the log, its corresponding PHYSUM and PHYCNT entries are reset to zero, and collection begins anew.

\section{MODULES}

Modules are the entities that give APES its character. Any digital circuit simulation program requires both a set of wires and a set of devices to wire. In APES, these devices are conceptual modules. These modules have what can be thought of as syntax and semantics. The syntax consists of a list of connection points, and the semantics are a description of how the outputs change as a function of the inputs and other changes in the system. 
APES requires only one particular module in order to run a schedule. In fact, the designer can at system generation time add, remove, rename, or otherwise alter the modules that are available to the user.

In addition to the inputs and outputs and the semantics, some modules have other parameters. Param. eter values are requested automatically by APES when the schedule designer adds those modules to his configuration via the ADD MODULE command. They are therefore fixed in the configuration and will be present when the schedule is loaded into a box. However, an operator may use the SET PARAMETER command to alter the settings after he loads a box. Parameters may vary among different instances of each module type.

\section{ENVIRONMENT NEEDED FOR APES}

The following items are needed for APES to run: (1) LSI-11 or PDP-11, preferably with extended instruction set (EIS), 28K words of memory;(2) RT-11 singlejob operating system (from DEC); (3) dual RK-05 or larger hard-disk drives; (4) one or more parallel inter- face boards (DRV.11 or equivalent); (5) one homebuilt interrupt generator board for each parallel interface; (6) Colbourne (or equivalent) voltage converters, debouncing switch inputs, and contour following integrators for the A/D inputs; (7) real-time clock (KWV-11 or equivalent); (8) one A/D converter, 16-channel multiplexed.

\section{SUMMARY}

We have discussed the design and implementation details of a system for real-time control of a laboratory of experimental psychology. So far, the system has been used to monitor the drinking rate of rats and to control operant and physiological data collection in our monkey behavioral pharmacology laboratory. We are preparing to use it in our human behavioral pharmacology laboratory. We are currently using it in conjunction with a commercially available data management software system RS/1 (Bolt Beranek and Newman) to perform data retrieval, statistical analysis, transformations, and graphics. 